Activités

$18-2$ | 2021

Espace et temps de l'activité / Travail, Territoire et politique

\title{
Travail, Territoire et Politique
}

Introduction au dossier

Nadia Heddad

(2) OpenEdition

Édition électronique

URL : https://journals.openedition.org/activites/6920

DOI : $10.4000 /$ activites. 6920

ISSN : $1765-2723$

Éditeur

ARPACT - Association Recherches et Pratiques sur les ACTivités

\section{Référence électronique}

Nadia Heddad, «Travail, Territoire et Politique », Activités [En ligne], 18-2 | 2021, mis en ligne le 15 octobre 2021, consulté le 07 avril 2022. URL : http://journals.openedition.org/activites/6920 ; DOI : https://doi.org/10.4000/activites.6920

Ce document a été généré automatiquement le 7 avril 2022.

\section{(c) (i) (9)}

Activités est mis à disposition selon les termes de la licence Creative Commons Attribution - Pas d'Utilisation Commerciale - Pas de Modification 4.0 International. 


\title{
Travail, Territoire et Politique
}

\author{
Introduction au dossier
}

\author{
Nadia Heddad
}

1 Le terme « territoire » est ancien, il apparait au XII ${ }^{\mathrm{e}}$ siècle et fait référence à territorium qui indique en latin une «étendue sur laquelle vit un groupe humain » (p. 3804, Rey, 1998). La dimension juridique et politique arrive plus tard, vers le XVII ${ }^{\mathrm{e}}$. Le mot «territoire» est ensuite utilisé en éthologie au $\mathrm{XX}^{\mathrm{e}}$ siècle pour désigner un espace délimité du point de vue animal. Lui sont associés les mots «territorialité », « territorialiser » et « déterritorialiser » (Ibid.).

2 Selon Paquot (2009), le terme " territoire », tout comme le vocable " espace ", ont été utilisé avec parcimonie dans le passé. En faisant référence au géographe Vidal de la Blache (1845-1918), l'auteur indique que le mot « région » était plus largement employé et cela jusqu'à dans les années 1970. Il est ensuite remplacé par le vocale " espace » avant de laisser la place dans les années 1980 au terme «territoire », fortement mis en avant depuis. Le rapprochement entre les mots «territoire » et « espace » accentue la polysémie du terme territoire (Paquot, 2011). En effet l'origine du vocable « espace » est obscure (Cazamian \& Lautier, 1996). Il est abstrait.

3 Pour la géographe Maryvonne Le Berre (1995), « Le territoire peut être défini comme la portion de la surface terrestre, appropriée par un groupe social pour assurer sa reproduction et la satisfaction de ses besoins vitaux. C'est une entité spatiale, le lieu de vie du groupe, indissociable de ce dernier.» (Paquot, 2009, p. 12). Pour Raffestin, cité par Paquot (Ibid.), « le territoire est un espace transformé par le travail humain ». Le mot territoire dessine ainsi un découpage "relié à l'action humaine " (p. 12, Ibid.). En proposant une unité de lecture qui ne soit pas que topographique, mais aussi reliée à ce que font les humains, un lien peut être esquissé avec l'ergonomie. En effet, bien que polysémique et évolutif dans le temps (Blanc, 2020 ; Paquot, 2009, 2011 ; Rey, 1998), le terme ouvre des perspectives pour la discipline, même s'il ne lui est pas familier. C'est ce que propose ce dossier d'explorer. 


\section{Évolution du travail et questions de territoire}

4 L'ergonomie de langue française défend l'idée de la centralité du travail comme «opérateur de santé» (Dejours, 2016), mais aussi de performance dans les organisations de production. Elle remet en cause l'approche par le poste de travail et propose la notion de situation de travail pour éclairer le périmètre dans lequel se déploie l'activité de travail. La situation de travail est posée comme une unité d'analyse pour saisir la dimension organisée de l'action dans la réalisation du travail réel. Elle permet d'opérer des liens à différentes échelles, celle micro de l'activité de travail réel avec celle plus macro des choix stratégiques des entreprises. L'ergonomie s'est ensuite élargie aux questions collectives et organisationnelles et interroge depuis peu les modèles économiques dans les entreprises.

5 Avec le déploiement important des services et les enjeux de développement durable, les situations de travail débordent les frontières classiques de l'entreprise. Elles sont aujourd'hui en prise avec des dimensions de l'ordre du territoire. Cette échelle est nouvelle pour la discipline.

6 Cet élargissement s'explique par l'intérêt que porte l'ergonomie au caractère singulier et situé de l'activité de travail. S'intéresser au territoire est d'une certaine manière, la poursuite de cet élargissement pour le périmètre d'une situation de travail qui concentre à elle seule une forme d'enchevêtrement des échelles.

7 Du macro initialement situé en ergonomie à l'échelle de l'entreprise, la situation de travail est aujourd'hui interpelée et construite tout comme elle y participe avec un niveau encore plus macro, celui du territoire. Le travail réel des salariés s'imbrique aux activités du vivant qu'il soit humain, animal ou végétal, du territoire avec lequel il constitue un tout. L'un influençant l'autre dans un assemblage dynamique, singulier et œuvrant nécessairement dans un temps long (Gaudart, 2014), celui de l'expérience et de l'historicité des personnes au travail en prise avec ce qui compose un territoire.

8 Le territoire contribue à organiser la manière dont le travail s'opère dans une organisation de travail tout comme le travail et l'organisation dans laquelle il est inscrit contribue à faire territoire. Autrement dit, le territoire peut être catalyseur comme il peut entraver le travail, mais il est aussi le résultat de la façon dont le travail vient investir et participer au territoire.

9 Cette interpellation contemporaine avec la dimension plus large du territoire conduit ainsi à s'intéresser à la situation de travail non pas dans un système dont les frontières semblent délimitées et connues, mais à l'interface et aux frontières d'échelles d'analyse entrelacées.

10 Le territoire comme un système qui dépasse l'organisation du travail et qui modifie le travail apparait comme un écosystème organisant tant l'activité de ceux engagés dans une production que celle d'un tiers : les clients, les usagers, les patients ou même les animaux ou les arbres d'un éleveur ou cultivateur. C'est l'échelle d'une action collective que chacun peut influencer dans les relations de coopération ou de tension. L'échelle du territoire participe à l'analyse du travail situé.

11 La question du territoire est également une nouvelle interpellation pour l'intervention en ergonomie qui s'est à l'origine, structurée autour des dynamiques de projet de transformation s'inscrivant et s'inspirant de la conception. L'intervention s'est attelée à viser l'organisation non seulement comme échelle d'analyse, mais d'action. Il est 
aujourd'hui possible de s'interroger sur la pertinence de réélaborer le dispositif en intervention pour engager plus directement des enjeux de territoire. La question des nouvelles modalités d'intervention pour viser, non seulement la conception ou l'organisation, mais aussi les dynamiques territoriales se pose ainsi. Est-ce qu'intervenir dans les organisations productives, comme les services ou l'agriculture par exemple, suppose nécessairement une action qui déborde les frontières classiques de l'organisation pour toucher le territoire? Dans ce cas, quel peut être le dispositif d'intervention? Ces questions sont nouvelles et la manière d'y répondre est à interroger et construire.

\section{Les contributions au dossier}

12 Ce dossier est issu de la réflexion engagée dans le cadre du séminaire annuel du pôle ergonomie de l'Université Paris 1 Panthéon Sorbonne réalisé en mai 2020, en période de crise sanitaire ${ }^{1}$. Le séminaire a visé à interroger et explorer l'échelle de territoire en lien avec la question du travail, mais aussi l'échelle politique au niveau institutionnel et des acteurs territoriaux. Les communications du séminaire «travail, territoire et politique » ont permis de discuter des corrélations entre la notion de territoire et la question du travail, même si le vocal « territoire » reste peu stabilisé.

Ce dossier est composé de quatre articles qui viennent enrichir les communications orales dont les enregistrements sont disponibles en libre accès sur le site de la médiathèque de l'université ${ }^{2}$.

L'article de Liliana Cunha et Marianne Lacomblez montre pourquoi et comment l'activité de travail contribue à produire le territoire. À partir d'une contribution dans le cadre d'un projet de refonte d'un réseau de transports publics en bus en zone rurale, les autrices montrent comment l'activité de travail participe concrètement au processus de territorialisation. Elles montrent l'écart de l'approche du territoire entre les concepteurs et les conducteurs. Pour les concepteurs, le territoire a une existence objective appréhendée à partir d'indicateurs normatifs (densité de la population, pôles générateurs de mobilité et matrices origine-destination des déplacements). Pour les conducteurs l'approche est centrée sur la vie quotidienne et sur les interactions entre les citoyens du territoire leur permettant de développer un savoir du territoire comme un milieu de vie et de travail. Le niveau "micro " opéré en analyse de l'activité de travail des chauffeurs et le niveau «maco » des décisions administratives territoriales sont à considérer ensemble, car ils interagissent dans une forme de dialectique. Dans l'étude de cas, les conducteurs compensent les inégalités d'accès aux transports en commun en déployant des stratégies pour réduire la distance d'accès des usagers au réseau de transport et élargir les limites des itinéraires. Le travail des conducteurs vient d'une certaine façon prendre en charge ce que la déclinaison locale des politiques publiques laisse de côté. Le territoire est alors un intermédiaire entre le niveau «macro » de ce qui est prévu en matière de service public et de droit à la mobilité et le niveau «micro» de l'activité des conducteurs qui vient prendre en charge les « résidus » pour compenser les inégalités d'accès. Le territoire est agi et reconstruit par l'activité de travail dont il est aussi une résultante.

15 À partir d'un cas exploratoire d'approvisionnement de cantines d'entreprise en produits locaux, Chloé Le Bail, Marianne Cerf et Gwenola Yannou-Le Bris proposent un cadre d'analyse en ergonomie pour saisir l'activité de travail dans un contexte de 
dynamique inter-organisationnelle. L'article s'intéresse aux évolutions du travail de relocalisation de systèmes alimentaires dans les territoires avec l'objectif de reconnecter l'agriculture à l'alimentation et ainsi répondre aux enjeux politiques, sociétaux et de développement durable. Les autrices soulignent l'élasticité de la définition du territoire selon les acteurs qui modifient le système en débordant ses frontières dans une dynamique collective de reconception des processus de coordination intra et inter-organisationnelle. Dans le même temps, elles distinguent trois échelles de coordinations : internes à l'organisation, inter-organisationnelles et au niveau institutionnel. Chaque niveau ayant une complexité propre aux réseaux d'acteurs, règles de fonctionnement et capacité à modifier et faire progresser les règles et les coordinations. Elles mettent en lumière le «travail de territorialisation » qui s'opère lors des reconceptions intra et inter-organisationnelles faisant par la même évoluer l'espace géographique culturel et/ou politique identifié comme territoire dans la représentation du durable et local par les acteurs. Ce travail de territorialisation est pour les autrices, une condition pour un système alimentaire territorialisé et son développement en lien étroit avec les acteurs, leurs besoins, volontés, contraintes et ressources. En proposant un cadre pour l'analyse de l'activité, cet article ouvre sur la question de l'intervention en ergonomie.

Le texte proposé par Jeanne Robert et Pascal Béguin s'inscrit dans le cadre d'un projet de développement local de relance et de revalorisation de la cueillette du tilleul dans les Baronnies provençales. À partir d'une ethnographie de la cueillette du tilleul, le texte conceptualise le faire milieu et met en lumière la manière dont un tissu local riche contribue à former un milieu. Pour les auteur.es, le travail de cueillette du tilleul passe par une mise en correspondance du divers maillant les activités de travail de cueillette, de commerce et de l'agriculture paysanne contribuant ainsi à mettre en correspondance des gestes de travail pour s'adapter aux spécificités du commerce, des fleurs et des arbres. La mise en œuvre de ce processus de production imbriqué permet de faire milieu tout en contribuant à créer de la valeur. En reliant dans le cours de l'activité, travail, sociabilité, sensibilité et développement économique, la cueillette du tilleul rassemble et réorganise dans un processus immanent permettant une mise en cohérence d'éléments hétérogènes. Au travers des façons de faire pour cueillir, tailler, conditionner, valoriser et vendre le tilleul, l'article montre comment la production et la commercialisation du tilleul reposent sur un travail de maillage des liens entre de nombreux acteurs avec une diversité d'enjeux et de manières de travailler permettant ainsi de vivre ensemble. Le milieu est de ce point de vue un effet du faire milieu qui repose sur l'exploration et l'invention dans l'activité de travail des façons de vivre au travers du tissage des liens entre acteurs du territoire et de la filière. Les auteur.es apportent également un éclairage sur la manière dont le "défilage» de ce milieu a conduit à la « la mort du tilleul» avec la dévaluation du travail de cueillette du tilleul observée dès les années 1960 et le délitement progressif des liens ayant engendré une rupture entre le travail des cueilleurs et leur existence. Enfin, l'article ouvre sur la question de l'action et interroge la perspective d'un projet de développement local. L'idée défendue est de soutenir les gestes qui permettent de faire milieu au travers d'une conception qui favorise le développement d'un maillage, un tissu vivant, qui émane et se forme dans et par l'action dont il ne peut être séparé. Il s'agit de permettre les mises en cohérence en vue des continuités nécessaires pour vivre ensemble (au travail et hors travail) sur un territoire qui déborde les frontières administratives du territoire du Parc naturel régional des Baronnies provençales. 
17 L'article de Laurent Van Belleghem traite de la pratique de l'intervention en ergonomie dans un contexte de mise à distance du travail et le recours massif aux techniques numériques en période de crise sanitaire chahutant ainsi profondément les repères dans l'exercice du métier d'ergonome. Privés de la proximité physique historiquement, une condition des coopérations entre acteurs engagées dans le cadre d'une intervention dans un écosystème local d'acteurs, l'auteur interroge ce que signifie de faire perdurer les relations qui permettent d'agir et produire collectivement à distance. Est-ce la disparition des territoires ou est-ce que le recours des nouvelles technologies, la visioconférence par exemple, conduit à produire de nouveaux territoires de travail? En permettant une production qui s'affranchit d'un ancrage géographiquement précis, des salariés ont pu modifier leur lieu de vie sans nécessairement changer d'emploi. Par le déploiement massif du télétravail, une forme d'extension des territoires propres au travail est observée bouleversant le travail, son contenu et sa réalisation. L'auteur propose de mettre à l'épreuve et explorer la déclinaison d'un geste professionnel relié à la technique utilisée habituellement dans les projets de conception. Il s'agit pour lui de décliner à distance le recours à la simulation du travail comme ressource permettant d'œuvrer et orienter l'action collective dans le cadre d'un écosystème local d'acteurs à distance. En exploitant les outils numériques de visioconférence, il explore un outillage méthodologique pour l'intervention en ergonomie. À partir d'études de cas réalisées en période de confinement, l'auteur relaye différentes expérimentations et ajustements techniques réalisés dans un cabinet de conseil ayant décidé de poursuivre ses missions à distance. L'article s'appuie également sur une expérience d'enseignement à distance dans le cadre d'une continuité pédagogique imposée ayant permis d'explorer la possibilité de faire simuler à distance et explorer les questions d'activité de travail. Même s'il souligne l'importance d'un ancrage physique d'un écosystème de relations, l'auteur met l'accent sur les perspectives qu'offrent ces nouvelles techniques dans la manière dont elles peuvent œuvrer dans la mise en relation, recomposant ce faisant les territoires du travail.

\section{BIBLIOGRAPHIE}

Blanc, Y. (2020). Valeur et territoire. [Conférence] Séminaire annuel du pôle ergonomie de l'universitéé Paris 1, 25-27 mai 2020, Paris, France.

Dejours, C. (2016). Situations du travail. Paris : PUF.

Cazamian, P., \& Lautier, F. (1996). Les concepts de temps et d'espace. Leur évolution historique. In

P. Cazamian, F. Hubault, \& M. Noulin (Eds.), Traité d'ergonomie. Nouvelle édition actualisée (pp. 461-464). Toulouse : Octarès.

Gaudart, C. (2014). Les relations entre l'âge et le travail comme problème temporel. Perspectives interdisciplinaires sur le travail et la santé [En ligne], 16-1 | 2014. URL : http://

journals.openedition.org/pistes/3052; DOI : https://doi.org/10.4000/pistes.3052 
Le Berre, M. (1995). « Territoire ». In A. Bailly, R. Ferras, \& D. Pumain (Eds.), Encyclopédie de géographie. Economica.

Paquot, T. (2009). 1 : Qu'appelle-t-on un territoire ? In Th. Paquot (Ed.), Le territoire des philosophes, pp. 9-27). La Découverte. https://doi.org/10.3917/dec.paquo.2009.01.0009

Paquot, T. (2011). Qu'est-ce qu'un « territoire » ? Vie sociale, 2, 23-32. https://doi.org/10.3917/

vsoc.112.0023

Rey, A. (1998). Dictionnaire historique de la langue française. Le Robert.

\section{NOTES}

1. Le choix du thème a été arrêté avant la pandémie. Il a été décidé de maintenir le séminaire sous un format alors inédit d'un webinaire aujourd'hui, accessible en ligne. En chahutant les modes de travail, la crise sanitaire a actualisé tout en l'exacerbant l'interrogation du rapport du travail à la question du territoire.

2. Le séminaire a été organisé sur 3 matinées. Les contributions ont été :

- Blanc, Y., (2020, 28 mai). "Valeur et territoire». [Conférence]. Séminaire annuel du pôle ergonomie. Territoire, travail et politique. https://mediatheque.univ-paris1.fr/video/2509seminaire-dergonomie-2020-travail-territoire-et-politique-25-mai/

- Perdrigeat, J. (2020, 28 mai). "Implication citoyenne, management des services et travail des élus». [Conférence]. Séminaire annuel du pôle ergonomie. Territoire, travail et politique. https:// mediatheque.univ-paris1.fr/video/2509-seminaire-dergonomie-2020-travail-territoire-et-

politique-25-mai/

- Du Tertre, C. (2020, 28 mai). «Transitions et émancipations par le Travail ». [Conférence]. Séminaire annuel du pôle ergonomie. Territoire, travail et politique. https://mediatheque.univ-paris1.fr/ video/2509-seminaire-dergonomie-2020-travail-territoire-et-politique-25-mai/

Cerf, M., et Le Bail, C. (2020, 26 mai). Quelle approche ergonomique d'une alimentation durable au travail sur le plateau de Saclay? [Conférence]. Séminaire annuel du pôle ergonomie. Territoire, travail et politique. https://mediatheque.univ-paris1.fr/video/2503-seminaire-dergonomie-2020travail-territoire-et-politique-26-mai/

- Robert, R., (2020, 26 mai). Proposition pour une ethnographie du travail du tilleul dans les Baronnies provençale. Passer du territoire au milieu pour penser l'espace du travail: un enjeu pour la santé. [Conférence]. Séminaire annuel du pôle ergonomie. Territoire, travail et politique. https:// mediatheque.univ-paris1.fr/video/2503-seminaire-dergonomie-2020-travail-territoire-et-

politique-26-mai/

- Demissy, R. (2020, 26 mai). Territoire et patrimoine immatériel : interroger le rapport entre travail et modèle économique de développement. [Conférence]. Séminaire annuel du pôle ergonomie. Territoire, travail et politique. https://mediatheque.univ-paris1.fr/video/2503-seminairedergonomie-2020-travail-territoire-et-politique-26-mai/

- Denis, C. (2020, 27 mai). Ergonomie et pandémie : quel positionnement de l'ergonome interne en milieu hospitalier? [Conférence]. Séminaire annuel du pôle ergonomie. Territoire, travail et politique. https://mediatheque.univ-paris1.fr/video/2510-seminaire-dergonomie-2020-travail-territoire-

et-politique-27-mai/

- Brousse, E. (2020, 27 mai). Travail et pandémie en milieu hospitalier. [Conférence]. Séminaire annuel du pôle ergonomie. Territoire, travail et politique. https://mediatheque.univ-paris1.fr/video/ 2510-seminaire-dergonomie-2020-travail-territoire-et-politique-27-mai/

- Cayon, C.. (2020, 27 mai). Responsable Prévention Risques Professionnels. MSA. Quelle stratégie d'intervention en santé, sécurité au travail en milieu agricole? [Conférence]. Séminaire annuel 
du pôle ergonomie. Territoire, travail et politique. https://mediatheque.univ-paris1.fr/video/ 2510-seminaire-dergonomie-2020-travail-territoire-et-politique-27-mai/

- Zittel, B. 2020, 27 mai). Réseau ergonomes Solidaires. [Conférence]. Séminaire annuel du pôle ergonomie. Territoire, travail et politique. https://mediatheque.univ-paris1.fr/video/2510seminaire-dergonomie-2020-travail-territoire-et-politique-27-mai/

- Mazzola, V. et Allain, A. (2020, 27 mai). Un atelier à distance pour l'aménagement de stations de télétravail. Séminaire annuel $\mathrm{du}$ pôle ergonomie. Territoire, travail et politique. https:// mediatheque.univ-paris1.fr/video/2510-seminaire-dergonomie-2020-travail-territoire-etpolitique-27-mai/

- Van Belleghem, L. (2020, 27 mai). Le masque, l'organisation, l'activité: l'épreuve d'une ergonomie à distance. [Conférence]. [Conférence]. Séminaire annuel du pôle ergonomie. Territoire, travail et politique. https://mediatheque.univ-paris1.fr/video/2510-seminaire-dergonomie-2020-travailterritoire-et-politique-27-mai/

\section{AUTEUR}

\section{NADIA HEDDAD}

Pôle ergonomie. FCPS. Université Paris 1. Panthéon Sorbonne - nadia.heddad@icloud.com 